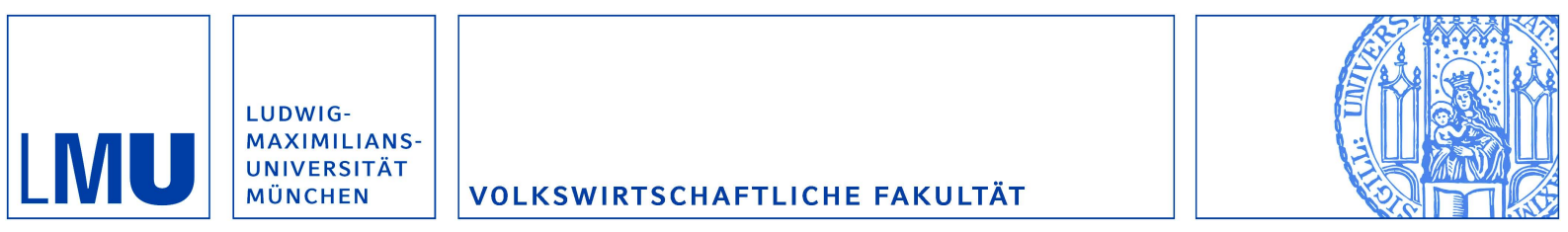

Kifmann, Mathias und Roeder, Kerstin:

The Political Sustainability of a Basic Income Scheme and Social Health Insurance

Munich Discussion Paper No. 2014-45

Department of Economics

University of Munich

Volkswirtschaftliche Fakultät

Ludwig-Maximilians-Universitäł München

Online at https://doi.org/10.5282/ubm/epub.21601 


\title{
The Political Sustainability of a Basic Income Scheme and Social Health Insurance
}

\author{
Mathias Kifmann* Kerstin Roeder ${ }^{\dagger}$
}

September 6, 2014

\begin{abstract}
This paper studies how society votes on the payroll taxes of a basic income and a social health insurance scheme. Individuals differ along the two most important dimensions when it comes to the design of the two welfare schemes, namely, income and risk. Even though the introduction of a basic income scheme opens up the possibility for additional redistribution, it also crowds out social health insurance. We show that when both welfare schemes are open for debate, the political equilibrium is such that only the basic income scheme prevails. At the constitutional stage we determine which welfare scheme society agrees to implement behind the veil of ignorance and with a Rawlsian objective. Since social health insurance not only redistributes income from rich to poor but also from low-risk to high-risk agents, the doubly disadvantaged in society - low-income and high-risk agents - may lose out in the political process when a basic income scheme is in place. Depending on the amount of health care expenditure and the inequalities in income and risk, it may well be that a society will find it optimal to set up an institutional framework for a social health insurance scheme only.
\end{abstract}

JEL-Classification: D6, D7, H1, H2, H5

Keywords: Basic Income, Social Health Insurance, Income Taxation, Political Support

\footnotetext{
${ }^{*}$ University of Hamburg, Department of Socioeconomics and Hamburg Center for Health Economics, Esplanade 36, 20249 Hamburg, Germany. Email: Mathias.Kifmann@wiso.uni-hamburg.de, Phone: +49-40-42838-4275.

${ }^{\dagger}$ Corresponding author. LMU Munich, Department of Economics, Ludwigstr. 28, 80539 Munich, Germany. Email: Kerstin.Roeder@lrz.uni-muenchen.de, Phone: +49-89-2180-1392.
} 


\section{Introduction}

Many policy makers and intellectuals have discussed the pros and cons of a basic income (for an overview see Clark and Kavanagh, 1996). A basic income (hereafter BI) is a governmentprovided cash payment that is paid unconditionally to all citizens on an individual basis without a means test or work requirement. It is a lifelong, guaranteed payment which, as is postulated by most proponents, should be large enough to satisfy an individual's basic needs. Various synonyms for a BI have entered the political discussion, for instance, state bonus, social dividend, guaranteed income, citizen's wage, citizenship income, existence income, or universal grant. The most frequently mentioned method of funding a BI scheme is a flat income tax (see, e.g., Atkinson, 1996b). Other similar policy measures that all share the idea of providing a "guaranteed income" to every citizen have been revisited, for instance, Milton Friedman's negative income tax (Friedman, 1962), or Tobin's demogrant (Tobin, Pechman and Mieszkowski, 1967). ${ }^{1}$

The philosopher and political economist Philippe Van Parijs is one of the most prominent advocates of a BI scheme of our time. ${ }^{2}$ He and his supporters consider such a scheme to be an effective and equitable solution to poverty, which not only promotes individual freedom but also leaves the beneficial aspects of a market economy in place (Van Parijs, 2004). It was only recently, in January 2014, that the European Citizens Initiative collected 285,042 statements of support for a basic scheme within the European Union. ${ }^{3}$ Even though this number of signatures is too low to prompt the European Commission to examine the initiative carefully and arrange for a public hearing in the European Parliament, the initiative shows that the idea of a basic income is part of an ongoing and lively debate on the future of the welfare state. So far, however, no country has ever introduced a full basic income scheme. ${ }^{4}$

At present, in most developed countries, income redistribution is attained through social insurance schemes, in particular through social health insurance which entitles citizens to health benefits when sick. Social health insurance (hereafter SHI) has a long history. The first scheme was introduced in Germany in 1883 as part of Bismarck's social insurance legislation. Since then, many countries have adopted similar schemes. They are typically financed by incomerelated contributions and thereby not only redistribute from those with a good health status to those with poor health status, but also from high-income to low-income earners. By combining these two dimensions of redistribution, SHI receives political support by two groups, those with

\footnotetext{
${ }^{1}$ For instance, under the negative income tax system, each person with an income below a certain threshold would receive supplemental pay from the government instead of paying taxes. See http://www.basicincome.org for an overview of the different ideas.

${ }^{2}$ In the 1980s, supporters of such a scheme set up the Basic Income European Network (BIEN) which publishes a regular newsletter and organizes conferences every two years.

${ }^{3}$ For further information see http://basicincome2013.eu/en/index.html.

${ }^{4}$ Alaska has a partial basic income scheme. Since 1982 everyone who has been officially resident in Alaska for at least six months is considered to be a part owner of the state's oil resources and receives a uniform dividend (in 2013: \$900) from the Alaska Permanent Fund every year; see http://www.apfc.org.
} 
poor health status and those with low income. As individuals with poor health status can be expected to be in a minority, support by the financially poor can be crucial for the existence of SHI if public provision is not exclusive (see Kifmann, 2005; and Nuscheler and Roeder, 2013 for an application to long-term care).

Most advocates of the basic income scheme regard it as complementary to social health insurance (see, e.g., Atkinson, 1996a; and Van Parijs, 1992) while others envision it as a substitution. From a political economy point of view, however, the crucial question is whether both incomeredistributing systems can receive political support, or if society should opts to rely solely on an SHI, or solely on a BI scheme. In particular, the introduction of a BI scheme could undermine support for SHI and the political process could create an outcome which was worse than the present situation with solely SHI. In such a case, society would do well to preclude a BI scheme in the first place.

In this paper we study this question in greater detail. We consider an economic environment where individuals differ in the two most important dimensions when it comes to the design of the two welfare schemes, namely income and health risk. A private health insurance market exists where individuals can buy health insurance coverage at an actuarial fair price. We consider Atkinson's (1996b) proposal that studying political support for the introduction of a BI scheme requires the analysis of two stages of decision making. First, at the constitutional stage, society votes on whether an SHI and/or a BI scheme should exist. In the second stage, this choice is followed by specification of the size of the prevailing scheme(s). At the constitutional stage individuals anticipate the possible voting outcomes at the second stage, but they are assumed to adjudicate as if they were behind a "veil of ignorance" about their own preferences. In other words, the decision on which welfare scheme(s) will be implemented is made in a state of uncertainty when individuals do not yet know their income and risk type. We apply the Difference Principle of Rawls (1971) and assume that individuals will then unanimously agree on the choice which maximizes the utility of the worst-off individuals in society, namely, the low-income and high-risk agents. ${ }^{5}$

Like Casamatta, Cremer and Pestieau (2000), who study the redistributive design of social insurance systems, we use the constitutional approach to capture the difference between the basic design of welfare state institutions and the decisions made within this framework. At the constitutional stage, decisions are made with a long-term perspective that includes fundamental decisions about the welfare institutions. At this stage, it is not yet clear whether individuals will be net payers into or receivers from the welfare state. The size of welfare schemes, on the other hand, is a more frequently debated issue and is settled through the political process. At this second stage individuals have learned their income and risk type and know whether they will be

\footnotetext{
${ }^{5}$ Analyzing the introduction of a BI scheme by applying Rawl's Difference Principle is also suggested by Van Parijs (1991).
} 
net payers into or beneficiaries of the welfare scheme(s) in place. The political process is thus governed by the concrete interests of members of society, which may differ from the objectives of the constitutional stage.

In our political process we let individuals vote on the flat income tax rate which finances the basic income scheme and/or on the SHI contribution rate financing the level of public health care benefits. Atkinson (1996b) suggests assessing the political feasibility of a BI scheme by the median voter model. However, as is well known, the median voter theorem may not apply in multi-dimensional issue spaces since preferences may not be single-peaked. To tackle this problem, we invoke Shepsle's (1979) concept of structure-induced equilibria in which individuals vote simultaneously but separately on each system. This allows us to extend the median voter approach to a two-dimensional policy space. Specifically, we identify a median voter for each issue. A political equilibrium is defined by the mutual best response of each median voter to the other median voter's preferred policy.

Our key finding is that when both welfare schemes are open for debate, the BI scheme completely crowds out SHI. In the second stage voting game, only the BI scheme prevails. Its size is determined by the median voter's income relative to average income. This result is in line with Meltzer and Richard (1981), who show that in democracies, more unequal income distributions induce wider redistribution policies. Depending on the inequality in health and income and on the extent of health care expenditure, we find that society may want to abandon a BI scheme at the constitutional stage and instead rely solely on SHI. The intuition for this result is that SHI is desirable from an ex-ante point of view because it not only redistributes from the rich to the poor but additionally from the healthy to the sick.

The paper is structured as follows. In Section 2, we present the model economy and the equilibrium concept. Section 3 first determines the median voters of the two policy issues at stake and then analyzes the political economic equilibrium. Decisions at the constitutional stage are analyzed in Section 4. In Section 5 we provide a numerical example. Finally, Section 6 provides some concluding remarks.

\section{The Model Economy}

\subsection{The Setup}

Individuals differ in two dimensions, namely in income $y_{i} \in \mathbb{R}^{+}$and in the probability of falling ill $\pi_{j} \in(0,1)$. They can either be poor or rich $i=p, r$ with $y_{p}<y_{r}$ and have either a low or a high probability of falling ill $j=l, h$ with $\pi_{l}<\pi_{h}$. Population size is normalized to one and the proportion of type- $i j$ individuals is given by $\theta_{i j}$. To ease notation, we write $\theta_{i}=\sum_{j} \theta_{i j}$ for the share of income type $i$ and $\theta_{j}=\sum_{i} \theta_{i j}$ for the share of risk type $j$. In the following, we assume 
Table 1: Proportions of agents in society.

\begin{tabular}{|c|c|c|c|c|}
\hline & \multicolumn{2}{|c|}{ Income } & \multirow[b]{2}{*}{$\Sigma$} \\
\hline & & $y_{p}$ & $y_{r}$ & \\
\hline \multirow{3}{*}{ Health risk } & $\pi_{l}$ & $\theta_{p l}$ & $\theta_{r l}$ & $>0.5$ \\
\hline & $\pi_{h}$ & $\theta_{p h}$ & $\theta_{r h}$ & $<0.5$ \\
\hline & $\Sigma$ & $>0.5$ & $<0.5$ & 1 \\
\hline
\end{tabular}

$\theta_{p}>0.5$ implying that median income is below average income: $y^{m}=y_{p}<\bar{y} \equiv \theta_{p} y_{p}+\theta_{r} y_{r}$. Additionally, the share of $l$-types is larger in society, implying $\theta_{l}>0.5$. Median risk is therefore below average risk: $\pi^{m}=\pi_{l}<\bar{\pi} \equiv \theta_{l} \pi_{l}+\theta_{h} \pi_{h} \cdot{ }^{6}$ None of the four groups comprises a majority in the population, i.e., $\theta_{i j} \in[0,0.5) \forall i j$. Table 1 summarizes.

Individuals derive utility from consumption of a numeraire commodity $c$. If ill, individuals require treatment leading to medical expenditure $M \in \mathbb{R}^{+}$. The SHI insures a share $\alpha \in[0,1]$ of health care expenditure. To finance social insurance, the contribution rate $s$ is applied to each individual's income. The system is not exclusive and additional health insurance coverage can be bought in the private market at an actuarially fair price $\pi_{j}$ per unit of coverage $I$. That is, insurance companies are able to observe each individual's risk type and premiums are given by $\pi_{j} I$. In addition to SHI, a BI scheme paying each individual a lump-sum transfer $\tau$ can exist. These transfers are financed by a proportional tax $t$ on each individual's income.

\subsection{Individual Optimization}

Individuals determine their consumption levels and insurance coverage by maximizing expected utility subject to their budget constraints in the good (superscript ' $g$ ') and bad (superscript ' $b$ ') health state

$$
\begin{array}{rl}
\max _{c_{i j}^{g}, c_{i j}^{b}, I_{i j}} & E U_{i j}=\left(1-\pi_{j}\right) u\left(c_{i j}^{g}\right)+\pi_{j} u\left(c_{i j}^{b}\right) \\
\text { s.t. } & c_{i j}^{g}=(1-t-s) y_{i}+\tau-\pi_{j} I_{i j} \\
& c_{i j}^{b}=(1-t-s) y_{i}+\tau-\pi_{j} I_{i j}-M+I_{i j}+\alpha M,
\end{array}
$$

\footnotetext{
${ }^{6}$ In a study of the potentially privately insured in the United States, Pauly and Herring (2007) found that the median expected health care expenses were US\$1,461 whereas the average expected expenses were US\$1,817 (1996-2002, in 2002 US\$).
} 
where $u^{\prime}>0, u^{\prime \prime}<0$ and $\lim _{c \rightarrow 0} u^{\prime}(c)=\infty$. As private insurance premiums are actuarially fair, individuals fully insure, i.e., $I_{i j}^{*}=(1-\alpha) M \forall i j$; see Mossin (1968). ${ }^{7}$ This implies that consumption levels are equalized across health states, $c_{i j}^{g}=c_{i j}^{b} \equiv c_{i j} \forall i j$. The utility of individual $i j$ can then be written as

$$
E U_{i j}=U_{i j}=u\left((1-t-s) y_{i}+\tau-\pi_{j}(1-\alpha) M\right) \quad \forall i j .
$$

\subsection{The Welfare Schemes}

BI $\tau$ is financed from taxing labor income at rate $t$ while SHI coverage $\alpha M$ is financed from taxing labor income at rate $s$. Each welfare system is assumed to be individually balanced, so that its total expenditure has to be equal to the amount of collected taxes. ${ }^{8}$ We make use of the same approach as, for instance, Galasso and Profeta (2007) and Conde-Ruiz and Profeta (2007), in capturing the distortions due to income taxation by correcting the tax base with the distortionary factor $1-t-s$. This reduced form reflects the adverse impact of each transfer scheme on the labor supply decision. The budget constraints for the BI and the SHI scheme read as

$$
\begin{aligned}
& t(1-t-s) \bar{y}=\tau, \\
& s(1-t-s) \bar{y}=\alpha \bar{\pi} M .
\end{aligned}
$$

Note that the lump sum transfer $\tau$ displays a Laffer curve relationship with respect to the income tax rate and depends negatively on the SHI contribution rate. In fact, the SHI contribution rate induces a distortion which contributes to decreasing the average income in the economy and thus reduces tax benefits. Analogously, the share of health care expenditure insured by the government is governed by a Laffer curve relationship with respect to the SHI contribution rate and depends negatively on the income tax rate. The maximum of each Laffer curve is at $t^{\max }(s)=(1-s) / 2$ and $s^{\max }(t)=(1-t) / 2$, respectively. Assuming that the share $\alpha$ of health care publicly provided cannot exceed one, the SHI contribution rate required to balance the budget (6) satisfies

$$
s(t) \leq \hat{s}(t) \equiv \frac{1-t-\sqrt{(1-t)^{2}-4 \frac{\bar{\pi} M}{\bar{y}}}}{2} .
$$

\footnotetext{
${ }^{7}$ We assume that net income is always sufficiently high to finance insurance premiums $\pi_{j} I_{i j}^{*}$ implying $y_{p}>\pi_{h} M$.

${ }^{8}$ In most countries contributions to the social health insurance scheme are kept separate from other government mandated taxes and charges (see, Saltman, Busse and Figueras, 2004).
} 
If $(1-t)^{2}<4 \frac{\bar{\pi} M}{\bar{y}}$, the overall tax base is too small to finance all health care expenditure and the above constraint does not need to be considered. If $(1-t)^{2} \geq 4 \frac{\bar{\pi} M}{\bar{y}}$, we have

$$
\frac{\partial \hat{s}(t)}{\partial t}=\frac{\hat{s}(t)}{\sqrt{(1-t)^{2}-4 \bar{\pi} M / \bar{y}}}>0
$$

For a higher tax rate, the SHI contribution rate must increase to cover all health care expenditure because the tax base is smaller. Constraint (7) is thus more likely to be relevant for smaller values of $t$.

The government budget constraints (5) and (6) imply that only two of the four policy instruments, $(t, \tau, s, \alpha)$, can be freely set. We assume that society votes on the income tax rate $t$ and on the SHI contribution rate $s$. The lump sum transfer $\tau$ and the share of health care expenditure insured by the government $\alpha$ are then residually determined through equations (5) and (6).

\subsection{The Sequence of Events}

We consider the following sequence of events:

(1) Constitutional stage

Behind the veil of ignorance individuals decide on whether to implement an SHI scheme and/or a BI scheme.

(2) Political Process

(2a) Individual income $y_{i}$ and the probability of illness $\pi_{j}$ are revealed.

(2b) Individuals vote on

$-s$ if only SHI has been implemented

$-t$ if only BI has been implemented

$-s$ and $t$ if both schemes have been implemented.

At stage (1) individuals anticipate the outcome of stage (2). We therefore solve this game by backward induction. In Section 3, we analyze stage (2) focusing on the case where individuals vote on both $s$ and $t$. The two other cases follow from straightforward calculations. In Section 4, we take an ex-ante point of view and determine at the constitutional stage (1) which welfare scheme(s) society chooses to implement. Before proceeding, we first define the equilibrium notions for our economy. 


\subsection{The Economic Equilibrium}

The economic equilibrium can be defined as follows:

\section{Definition 1 (Economic equilibrium)}

For a given income tax and social contribution rate $(t, s) \in[0,0.5] \times[0,0.5]$, an economic equilibrium is an allocation $\left\{c^{g}\left(y_{i}, \pi_{j}\right), c^{b}\left(y_{i}, \pi_{j}\right), I\left(y_{i}, \pi_{j}\right)\right\}_{i=p, r}^{j=l, h}$ such that the following conditions hold:

(i) the consumer problem is solved; that is, agents maximize $E U_{i j}$ with respect to $c_{i j}^{g}, c_{i j}^{b}$ and $I_{i j}$ subject to equations (2) and (3);

(ii) the government's budget constraints are balanced, that is, equations (5) and (6) are satisfied; and

(iii) the private health insurance market and the market for the numeraire commodity are competitive and clear.

Substituting from (5) and (6) into (4), the utility level obtained in an economic equilibrium by a type-i $i$ individual is given by his indirect utility function

$$
V_{i j}(t, s)=u\left((1-t-s) y_{i}+t(1-t-s) \bar{y}-\pi_{j} M+\eta_{j} s(1-t-s) \bar{y}\right) \quad \forall i j,
$$

where $\eta_{j} \equiv \pi_{j} / \bar{\pi}$ is the ratio of individual to average risk. The above indirect utility function can be used to express the preferences of an individual of type $i j$ for the income tax rate $t$ and the SHI contribution rate $s$ in an economic equilibrium. Both policy variables are specified in the political process described in Section 3 .

\subsection{Structure-Induced Equilibrium}

In the political process, individuals vote for the income tax rate, $t$, and/or the SHI contribution rate, $s$. We assume that agents vote sincerely and that every voter has zero mass. The latter implies that no individual vote can change the outcome. We follow Shepsle (1979) in analyzing structure-induced equilibria, in which agents vote simultaneously, but separately on the issues at stake. Specifically, the political system we adopt has the following characteristics: ${ }^{9}$

(i) Preferences of the electorate, i.e., $p l-, p h-, r l-$, and $r h$-agents, are perfectly represented by the government which delegates policy issues to (perfectly representative) ministries: the Ministry of Health and the Ministry of Finance.

\footnotetext{
${ }^{9}$ The description of the structure-induced equilibrium closely follows Galasso (2008).
} 
(ii) The Ministry of Health is responsible for SHI while the Ministry of Finance is responsible for income taxation. In the political process, the Ministry of Health proposes an SHI contribution rate for a given income tax rate $s(t)$ which the Ministry of Finance can either accept or reject. Similarly, the Ministry of Finance suggests an income tax rate for a given SHI contribution rate $t(s)$ which, in turn, the Ministry of Health can either accept or reject.

(iii) Both ministries thereby act in the interests of the median voter of the respective policy issue.

Within this political system, the proposals $t(s)$ and $s(t)$ can be thought of as the best responses (or reaction functions) of the ministries that are rooted in the preferences of the median voter on the issue at stake. Their intersection characterizes the structure-induced equilibrium of the voting game, where policy proposals of the ministries are mutual best responses to one another. Thus, the structure-induced equilibrium introduces issue-by-issue voting and thereby allows the application of the median voter approach in a two-dimensional issue space.

This equilibrium concept also nests the voting outcome for the case where only one welfare scheme is in place. The size of the BI and SHI scheme is then simply determined by $t(0)$ and $s(0)$, respectively.

\section{Politico-Economic Equilibria}

In this section, we analyze the voting game. First, we compute every voter's ideal income tax rate for a given SHI contribution rate, $t(s)$, and then the ideal SHI contribution rate for a given income tax rate, $s(t)$. For each value of $s$, we then identify the median voter over $t$, and for each value of $t$, we verify the median voter over $s$.

\subsection{Voting on the Income Tax Rate}

For a given social insurance rate, $s$, a type- $i j$ individual chooses the preferred income tax rate $t_{i j}(s)$ by maximizing his indirect utility, equation (9), with respect to $t$ :

$$
\max _{t} V_{i j}(t, s) \quad \text { s.t. } \quad t \geq 0
$$

The first order condition (FOC) of this problem is

$$
\frac{\partial V_{i j}(t, s)}{\partial t}=u^{\prime}\left(c_{i j}\right)\left[-y_{i}+\left(1-2 t^{*}-s\right) \bar{y}-\eta_{j} s \bar{y}\right] \leq 0, \quad t^{*} \geq 0, \quad t^{*} \frac{\partial V_{i j}(t, s)}{\partial t}=0 .
$$


The first expression in brackets represents the direct costs of higher income taxes which are increasing in income. The second term reflects the increase in the lump-sum transfer, while the last expression captures the adverse effect of higher income taxes on the SHI scheme. As the indirect utility function of a type-ij agent is concave in $t$,

$$
\frac{\partial^{2} V_{i j}(t, s)}{\partial t^{2}}=u^{\prime \prime}\left(c_{i j}\right)\left[-y_{i}+(1-2 t-s) \bar{y}-\eta_{j} s \bar{y}\right]^{2}-u^{\prime}\left(c_{i j}\right) 2 \bar{y}<0 \quad \forall i j,
$$

preferences are single-peaked. Solving equation (10) for the income tax rate yields

$$
t_{i j}^{*}(s)=\max \left\{0 ; \frac{1-\omega_{i}-\left(1+\eta_{j}\right) s}{2}\right\}
$$

where we define $\omega_{i} \equiv y_{i} / \bar{y}$ as the ratio of individual to average income. The following Lemma summarizes the main properties of $t_{i j}^{*}(s)$ :

\section{Lemma 1 (Properties: income tax rate)}

The preferred income tax rate is zero for rich individuals, $t_{r l}^{*}(s)=t_{r h}^{*}(s)=0 \forall s$. Poor agents prefer positive income taxation when no SHI is in place, i.e., $t_{p j}^{*}(0)>0$. As long as $t_{p j}^{*}(s)>0$, the preferred income tax rate

(i) decreases in the SHI contribution rate, $\mathrm{d} t_{p j}^{*}(s) / \mathrm{d} s<0$, and

(ii) decreases in the probability of falling ill, $\mathrm{d} t_{p j}^{*}(s) / \mathrm{d} \pi_{j}<0$.

Unsurprisingly, rich agents oppose a positive income tax rate as they are net contributors to the welfare scheme. Poor agents, by contrast, gain from the income redistribution that the basic income tax scheme achieves through a combination of a proportional income tax rate and a lump-sum transfer, $\tau$. With no SHI, these agents always vote for positive taxation. For larger values of $s$, poor agents have to weigh the negative effect of income taxation on the tax base of the welfare schemes against its positive redistributive effects. High-risk, low-income individuals value this negative effect more as they are not only net beneficiaries from a BI but also from an SHI. Therefore, they prefer a lower income tax rate for any given SHI contribution rate.

It is now straightforward to order every individual's vote over the proportional income tax for a given SHI contribution rate, and to identify the median voter's type. This is the type-ij agent who divides the electorate into halves.

\section{Lemma 2 (Median voter: income tax rate)}

The most preferred income tax rates can be ordered as follows: $t_{r l}^{*}(s)=t_{r h}^{*}(s)=0 \leq t_{p h}^{*}(s) \leq$ $t_{p l}^{*}(s)$. Since the poor comprise a majority, the median voter is a poor high-risk type implying $t^{m}(s) \equiv t_{p h}^{*}(s)$. 


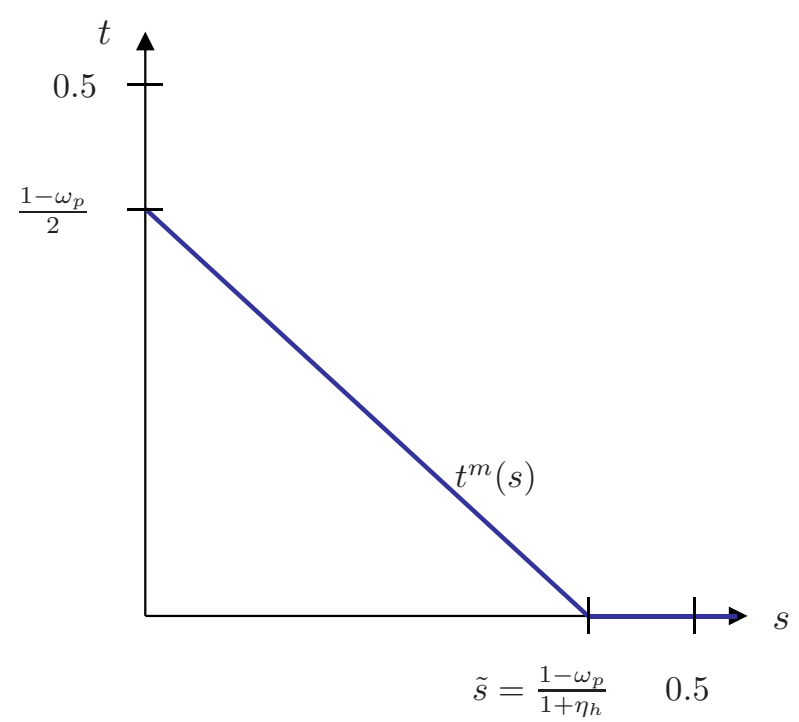

Figure 1: Median voter's preferred income tax rate.

The median voter's income tax reaction function, $t^{m}(s)$, can be written as

$$
t^{m}(s)=\max \left\{0, \frac{1-\omega_{p}-\left(1+\eta_{h}\right) s}{2}\right\}
$$

It is displayed in Figure 1. For no social insurance scheme, $s=0$, equation (12) reduces to

$$
t^{m}(0)=\frac{1-\omega_{p}}{2}>0
$$

as $\omega_{p}=y_{p} / \bar{y}<1$. In other words, if no SHI scheme exists, the median voter always votes for positive income taxation. As he is a poor agent, he pays less into the BI scheme than he gets out of it. Voting for positive income taxation thus increases his consumption possibilities. Moreover, we find a critical level of the SHI rate $\tilde{s}$ which induces a zero income tax rate:

$$
t^{m}(s)=0 \quad \text { for } \quad s \geq \tilde{s} \equiv \frac{1-\omega_{p}}{1+\eta_{h}}
$$

If the SHI contribution rate increase above $\tilde{s}$, distortions which come with SHI are too large and a BI scheme becomes unprofitable from the median voter's point of view.

\subsection{Voting on the SHI Contribution Rate}

For a given income tax rate, $t$, a type- $i j$ individual chooses his most preferred SHI contribution rate $s_{i j}(t)$ by maximizing his indirect utility, equation (9), with respect to $s$. Here the require- 
ment that health care expenditure cannot be refunded above 100 percent needs to be considered; see equation (7). ${ }^{10}$ Thus, the optimization problem of a type- $i j$ individual is:

$$
\max _{s} \quad V_{i j}(t, s) \quad \text { s.t. } \quad 0 \leq s \leq \hat{s}(t)
$$

The Kuhn-Tucker conditions of the above problem amount to

$$
\begin{array}{ll}
\frac{\partial V_{i j}(t, s)}{\partial s} \leq 0, \quad s^{*} \geq 0, & s^{*} \frac{\partial V_{i j}(t, s)}{\partial s}=0, \\
\frac{\partial V_{i j}(t, s)}{\partial s} \geq 0, & s^{*} \leq \hat{s}(t), \quad\left(\hat{s}(t)-s^{*}\right) \frac{\partial V_{i j}(t, s)}{\partial s}=0,
\end{array}
$$

where

$$
\frac{\partial V_{i j}(t, s)}{\partial s}=u^{\prime}\left(c_{i j}\right)\left[-y_{i}-t \bar{y}+\eta_{j}\left(1-t-2 s^{*}\right) \bar{y}\right]
$$

As for income taxation, the first term in brackets reflects the direct costs of higher health insurance contribution rates. The second expression captures their adverse effect on the tax base of the BI scheme, while the last term represents the benefit of higher SHI coverage which increases with the probability of falling ill. The indirect utility function is concave in $s$,

$$
\frac{\partial^{2} V_{i j}(t, s)}{\partial s^{2}}=u^{\prime \prime}\left(c_{i j}\right)\left(-y_{i}-t \bar{y}+\eta_{j}(1-t-2 s) \bar{y}\right)^{2}-u^{\prime}\left(c_{i j}\right) \eta_{j} 2 \bar{y}<0 \quad \forall i j,
$$

implying that preferences are single-peaked. We denote by $\check{s}_{i j}(t)$ the solution to equation $\partial V_{i j}(t, s) / \partial s=0$. From (17) we obtain

$$
\check{s}_{i j}(t)=\frac{\eta_{j}-\omega_{i}-\left(1+\eta_{j}\right) t}{2 \eta_{j}}
$$

The preferred SHI contribution rate of a type-ij individual can then be written as

$$
s_{i j}^{*}(t)=\max \left\{0 ; \min \left\{\check{s}_{i j}(t) ; \hat{s}(t)\right\}\right\} .
$$

The following Lemma summarizes the main properties of $s_{i j}^{*}(t)$ :

\section{Lemma 3 (Properties: SHI contribution rate)}

The most preferred SHI contribution rate is zero for rich low-risk individuals $s_{r l}^{*}(t)=0$. If $s_{i j}^{*}(t)=\check{s}_{i j}(t)>0$, the preferred $S H I$ contribution rate

(i) decreases in income, $\mathrm{d} s_{i j}^{*}(t) / \mathrm{d} y_{i}<0$,

\footnotetext{
${ }^{10}$ For high values of $t$, this constraint may be irrelevant because the tax base is too small to finance all health care expenditure.
} 
(ii) decreases in the income tax rate, $\mathrm{d} s_{i j}^{*}(t) / \mathrm{d} t<0$, and

(iii) increases in the probability of falling ill, $\mathrm{d} s_{i j}^{*}(t) / \mathrm{d} \pi_{j}>0$.

When the restriction that only 100 percent of health care expenditure can be refunded (i.e., $\alpha \leq 1)$ is non-binding, the results can be interpreted as follows. As the social health care system incorporates an element of income redistribution in that high-income agents pay more into the SHI scheme than low-income individuals, rich individuals vote for a lower SHI contribution rate than poor individuals. Moreover, a higher income tax rate adversely affects the overall tax base and reduces the attractiveness of SHI. High-risk individuals prefer a higher SHI contribution rate, unlike their low-risk counterparts, as they have to pay higher premiums in the private market while the SHI scheme redistributes from high- to low-risk agents.

With Lemma 3, the preferred SHI contribution rate can be ranked across agents and the median voter can be identified.

\section{Lemma 4 (Median voter: SHI contribution rate)}

The preferred SHI contribution rates for a given income tax rate, $s_{i j}^{*}(t)$, can be ranked according to $s_{r l}^{*}(t)=0 \leq s_{p l}^{*}(t), s_{r h}^{*}(t) \leq s_{p h}^{*}(t)$. Since neither high-risk nor rich agents form a majority, the median voter is a poor low-risk type.

The median voter's reaction function over the SHI contribution rate for a given income tax rate $s^{m}(t)$ is thus given by

$$
s^{m}(t)=\max \left\{0 ; \min \left\{\check{s}_{p l}(t) ; \hat{s}(t)\right\}\right\} .
$$

Noting that $\check{s}_{p l}^{\prime}(t)<0$ and $\hat{s}^{\prime}(t)>0$ (see equation $(8)$ ), a sufficient condition for $\check{s}_{p l}(t) \leq \hat{s}(t)$ is

$$
\check{s}_{p l}(0)=\frac{\eta_{l}-\omega_{p}}{2 \eta_{l}} \leq \hat{s}(0)=\frac{1-\sqrt{1-4 \frac{\bar{\pi} M}{\bar{y}}}}{2} .
$$

This is shown in Figure 2(a) (note that the axes are reversed). If $\hat{s}(0)<\check{s}_{p l}(0)$, the median voter's preferred SHI contribution rate has a kink. This is illustrated in Figure 2(b). For low values of $t$, he desires more redistribution than possible through SHI. Such a scenario is more likely if health care expenditure $\bar{\pi} M$ is small compared to average income $\bar{y}$.

In Figure 2(a), the preferred SHI contribution rate for no income taxation, $t=0$, equation (20) reduces to

$$
s^{m}(0)=\check{s}_{p l}(0)=\frac{\eta_{l}-\omega_{p}}{2 \eta_{l}} .
$$

The above equation is positive if $\eta_{l}>\omega_{p} . \eta_{l}=\pi_{l} / \bar{\pi}<1$ is a measure for the inequality in illness. The closer it is to one, the smaller the inequality in illness (for a given share of risk 


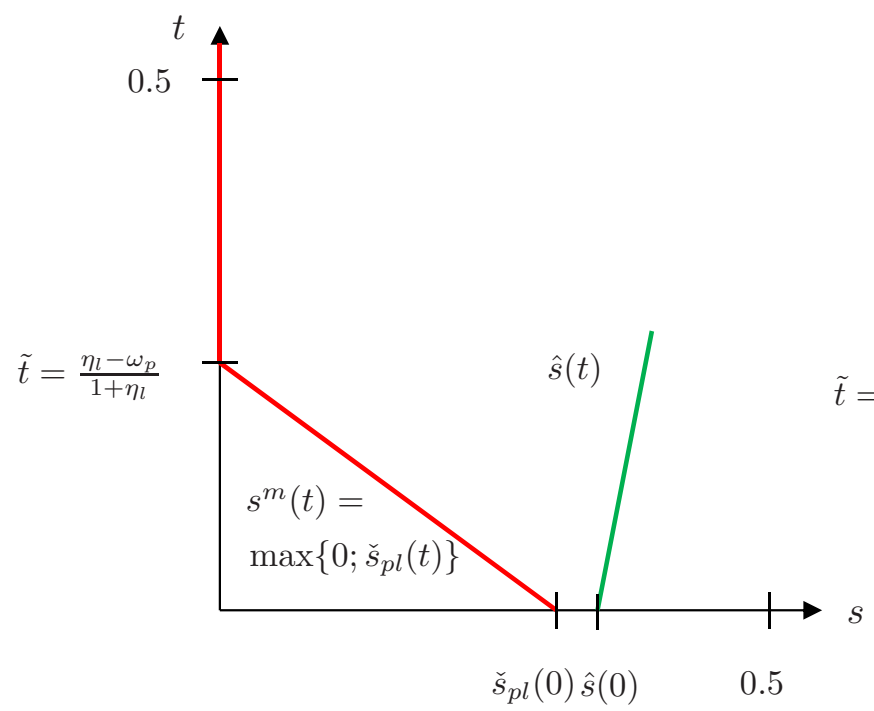

(a)

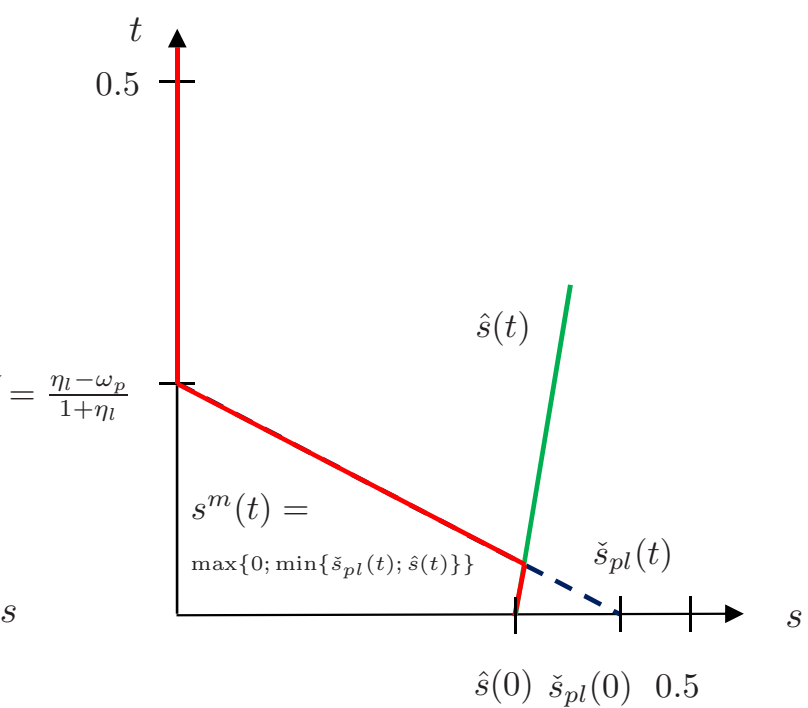

(b)

Figure 2: Median voter's preferred SHI contribution rate.

types). Likewise, $\omega_{p}=y_{p} / \bar{y}<1$ measures the inequality in income. The smaller $\omega_{p}$, the larger the income equality. The median voter thus prefers a positive SHI contribution rate only if the inequality in illness is smaller than the inequality in income. On the one hand, the median voter profits from income redistribution through SHI as he is in the lower income class. The extent to which he benefits from this redistribution decreases in $\omega_{p}$, that is, in his own income relative to average income. On the other hand, the median voter is a low-risk agent, which implies that he cross-subsidizes high-risk agents via SHI. This negative effect decreases in $\eta_{l}$ and therefore decreases in his own probability of needing health care relative to the average risk. Only, if the former (positive) effect outweighs the latter (negative) effect, does he vote for SHI.

In Figure 2(b), we obtain for the preferred SHI contribution rate and no income taxation, $t=0$,

$$
s^{m}(0)=\hat{s}(0)=\frac{1-\sqrt{1-4 \frac{\bar{\pi} M}{\bar{y}}}}{2} .
$$

In this case, the SHI system covers 100 percent of health care expenditure in the absence of a BI scheme. 
In both figures, we find a critical level of the income tax rate $\tilde{t}$ above which the SHI contribution rate becomes zero:

$$
s^{m}(t)=0 \quad \text { for } \quad t>\tilde{t} \equiv \frac{\eta_{l}-\omega_{p}}{1+\eta_{l}}
$$

\subsection{Equilibrium Outcomes}

In Sections 3.1 and 3.2, we analyzed the voters' decisions on the income tax rate and the SHI contribution rate. Both median voters were found to have low income, but the decisive voter over the income tax rate is a high-risk agent, whereas the one over the SHI contribution rate is a low-risk agent.

If the institutional framework is such that only one welfare scheme is in place, equation (12) determines the size of the BI scheme for $s=0$, and equation (20) determines the size of the SHI scheme for $t=0$. The corresponding equilibrium values are given by

$$
t^{I}=t^{m}(0)=\left(1-\omega_{p}\right) / 2
$$

and

$$
s^{I I}=s^{m}(0)=\max \left\{0 ; \min \left\{\check{s}_{p l}(0) ; \hat{s}(0)\right\}\right\} .
$$

When both welfare schemes are open for debate, equations (12) and (20) can be interpreted as reaction functions: for a given SHI contribution rate, equation (12) identifies the income tax rate chosen by the median voter $p h$; and for a given income tax rate, equation (20) identifies the SHI contribution rate chosen by the median voter $p l$. The structure-induced equilibrium outcomes of this voting game correspond to the points at which these two reaction functions cross. In the following, we will specify this structure-induced equilibrium.

If $\eta_{l} \leq \omega_{p}$, SHI receives no support. The equilibrium is $t^{I}=t^{m}(0)=\left(1-\omega_{p}\right) / 2$ and $s^{I}=0$. The case of $\eta_{l}>\omega_{p}$ is displayed in Figure 3. Note that $t^{m}(0) \geq \tilde{t}$, that is the reaction function $t^{m}(s)$ hits the $t$-axis above $\tilde{t}$, the critical level of the income tax rate above which the SHI contribution rate becomes zero. ${ }^{11}$ First, we consider the case in which the reaction function for the SHI contribution rate $s^{m}(t)$ does not display a kink, as in Figure 3(a). From (14) and (22), we obtain

$$
\check{s}_{p l}(0)<\tilde{s} \quad \Leftrightarrow \quad \frac{\eta_{l}-\omega_{p}}{2 \eta_{l}}<\frac{1-\omega_{p}}{1+\eta_{h}} \quad \Leftrightarrow \quad \omega_{p}\left(2 \eta_{l}-1-\eta_{h}\right)<\eta_{l}\left(1-\eta_{h}\right) .
$$

The last inequality holds because $\eta_{l}>\omega_{p}$ and $2 \eta_{l}-1-\eta_{h}<1-\eta_{h}$. The $t^{m}(s)$ function thus hits

\footnotetext{
${ }^{11}$ The function $t(a)=\left(a-\omega_{p}\right) /(1+a)$ is increasing in $a$.
} 


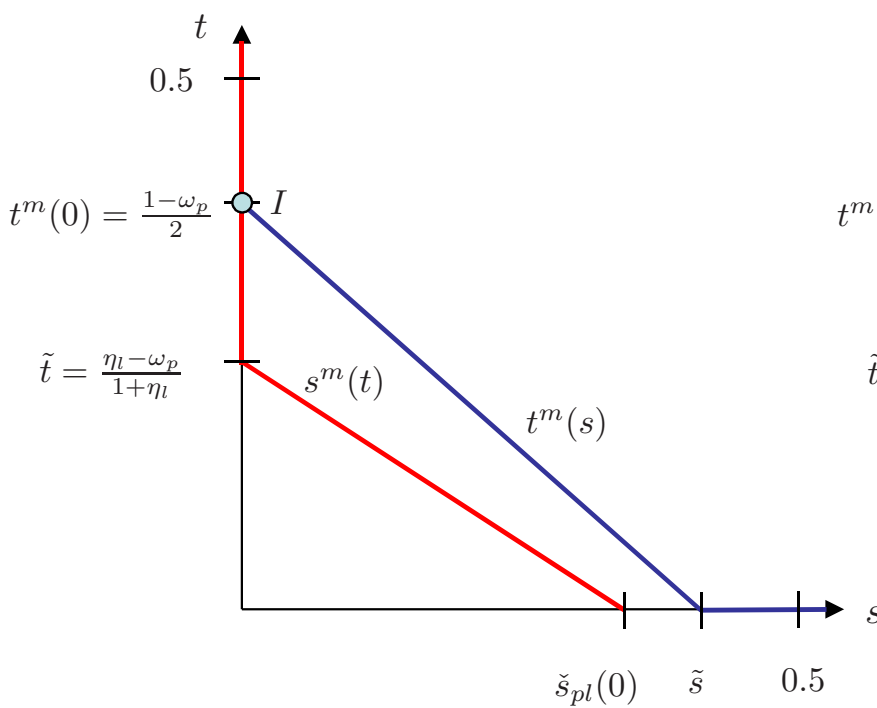

(a)

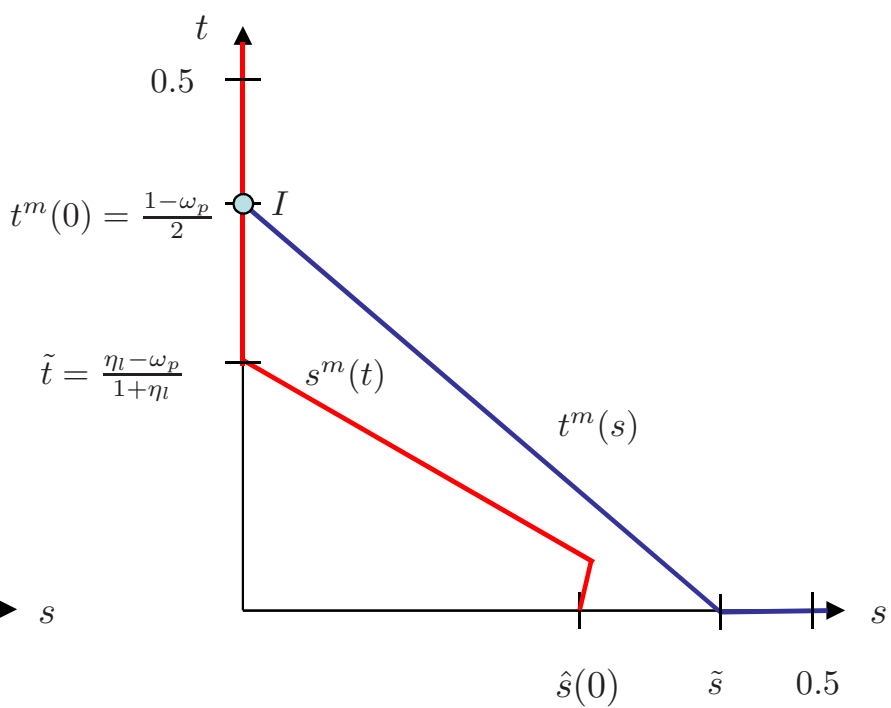

(b)

Figure 3: Political equilibria.

the $s$-axis to the right of $\check{s}_{p l}(0)$ implying that the two functions cross only once. The equilibrium is unique and is again characterized by

$$
t^{I}=\frac{1-\omega_{p}}{2} \quad \text { and } \quad s^{I}=0 .
$$

In words, only a BI scheme prevails. Figure 3(a) provides an illustration.

When the reaction function $s^{m}(t)$ for the SHI contribution rate displays a kink as in Figure $3(\mathrm{~b})$, the restriction that only 100 percent of health care expenditure can be refunded is binding for low levels of $t$. But since the reaction function $t^{m}(s)$ is always above the reaction function $s^{m}(t)$, this has no consequences for the political equilibrium, implying that the unique equilibrium remains $t^{I}$ and $s^{I}$. The following proposition summarizes our results.

\section{Proposition 1 (Political equilibrium)}

(i) If the institutional framework for only a BI scheme is in place, while the SHI scheme is abandoned at the constitutional stage $(s=0)$, the BI scheme's size is determined by

$$
t^{I}=t^{m}(0)=\left(1-\omega_{p}\right) / 2 .
$$

(ii) If the institutional setting for only an SHI scheme is in place, while the BI scheme is 
abandoned at the constitutional stage $(t=0)$, the SHI scheme's size is determined by

$$
s^{I I}=s^{m}(0)=\max \left\{0 ; \min \left\{\check{s}_{p l}(0) ; \hat{s}(0)\right\}\right\} .
$$

(iii) If the size of both welfare schemes is open for debate in the second stage, the SHI scheme finds no political support and only a BI scheme prevails. Specifically, the size of each welfare scheme is

$$
t^{I}=\frac{1-\omega_{p}}{2} \quad \text { and } \quad s^{I}=0
$$

Our analysis has shown that in the second stage voting game, coexistence of BI and SHI will never find political support. Once a BI scheme is open for debate in addition to SHI, the BI scheme completely crowds out SHI. The intuition for this result is that the median voter determining the SHI contribution rate is a poor low-risk agent who has only limited interest in SHI. Poor high-risk individuals are pivotal only for the BI scheme. Given their low income, they prefer a high level of BI. This level is so high that in the political equilibrium, poor low-risk agents have no more interest in SHI.

In the following section, we will analyze whether and, if so, under what conditions it might be optimal for society to abandon a BI in the first place and to set up the institutional framework for an SHI scheme only.

\section{Constitutional Stage}

In accordance with Atkinson (1996), we now turn to the constitutional stage in which it is decided whether a BI and/or an SHI system exist(s). Constitutional rules are made on an ex ante basis, that is before individuals know their income and risk type. Utility levels are evaluated at the second-stage voting equilibrium. We follow Rawls (1971) and assume that behind the veil of ignorance, individuals will unanimously agree on the choice that maximizes the utility of the worst-off individuals, i.e., the low-income high-risk agents. ${ }^{12}$

In our former analysis, we found that in a political equilibrium in the second stage voting game BI and SHI cannot coexist. The choice at the constitutional stage thus pins down to:

(i) setting up the institutional framework for solely a BI scheme with $t^{I}=\left(1-\omega_{p} / 2\right)$, or

(ii) implementing solely an SHI which comes with $s^{I I}=\max \left\{0 ; \min \left\{\check{s}_{p l}(0) ; \hat{s}(0)\right\}\right\}$.

\footnotetext{
${ }^{12}$ Alternatively, one can think of a benevolent social planner who determines the sort of rigid constitutional setting by maximizing utility of the worst-off. The political decisions about the size of the welfare scheme(s), on the other hand, are more frequently debated and reflect the interest of the median voter (see also Casamatta et al. 2000)
} 
Before proceeding with the constitutional choice, let us determine the optimal welfare design for the worst-off individuals - the high-risk poor - without political constraints. The problem corresponds to

$$
\max _{s, t} \quad V_{p h}(t, s)=u\left((1-t-s) y_{p}+t(1-t-s) \bar{y}-\pi_{h} M+\eta_{h} s(1-t-s) \bar{y}\right)
$$

subject to the constraints $t \geq 0$ and $0 \leq s \leq \hat{s}(t)$. The solution is (see Appendix A.1 for details)

$$
\begin{array}{lllrl}
t_{p h}^{\circ}=0 & \text { and } & s_{p h}^{\circ}=\min \left\{\check{s}_{p h}(0) ; \hat{s}(0)\right\}>0 & \text { if } & \hat{s}(0) \geq \frac{1-\omega_{p}}{1+\eta_{h}} \\
t_{p h}^{\circ}>0 & \text { and } & s_{p h}^{\circ}=\hat{s}\left(t_{p h}^{\circ}\right)>0 & \text { if } & \hat{s}(0)<\frac{1-\omega_{p}}{1+\eta_{h}} .
\end{array}
$$

Thus, an SHI is always optimal for $p h$-individuals. If $\hat{s}(0)$ is large, then type- $p h$ individuals' utility is maximized if only an SHI system is in place. Otherwise, if $\hat{s}(0)$ is small, that is, if coverage of all health care expenditure requires only a small SHI system, then SHI covers all health care expenditure, and a BI system exists on top. The SHI scheme has the advantage that it redistributes income in both dimensions relevant for $p h$ individuals. Only if all health care expenditure is covered, is additional income redistribution via a BI optimal.

In a political equilibrium, SHI will only be implemented if $\mathrm{BI}$ is ruled out at the constitutional stage. In this case, the SHI contribution rate is governed by the preferences of $p l$-individuals, who prefer a lower rate than $p h$-individuals. Nevertheless, low-income high-risk agents may be better off with a smaller SHI contribution rate rather than no SHI system. So, it could well be that, given that utility is evaluated at $t^{I}$ and $s^{I I}$, the utility of the doubly disadvantaged is maximized if society agrees to implement solely an SHI scheme at the constitutional stage.

To begin with, we focus on the case of $s^{I I}=\check{s}_{p l}(0)$ implying that health care is only partially covered in the political equilibrium without BI. Then $s^{I I}=\left(\eta_{l}-\omega_{p}\right) / 2 \eta_{l}$ and the indirect utility of type- $p h$ agents is given by

$$
V_{p h}^{I I}=u\left(\left(1-\frac{\eta_{l}-\omega_{p}}{2 \eta_{l}}\right)\left(y_{p}+\frac{\eta_{l}-\omega_{p}}{2} \frac{\eta_{h}}{\eta_{l}} \bar{y}\right)-\pi_{h} M\right) .
$$

In equilibrium $I$ with only a BI scheme, we have $t^{I}=\left(1-\omega_{p}\right) / 2$. Using (9), the indirect utility of a type-ph agent amounts to

$$
V_{p h}^{I}=u\left(\left(1-\frac{1-\omega_{p}}{2}\right)\left(y_{p}+\frac{1-\omega_{p}}{2} \bar{y}\right)-\pi_{h} M\right) .
$$

We have $s^{I I} \leq t^{I}$, that is, the level of income redistribution is lower in equilibrium $I I$ than in equilibrium $I .{ }^{13}$ Since, however, $p h$-types benefit from the cross-subsidization inherent in SHI,

\footnotetext{
${ }^{13}$ Note that $s^{I I}\left(\eta_{l}=1\right)=t^{I}$. Since $\frac{\partial s^{I I}}{\partial \eta_{l}}>0$ and $\eta_{l}<1$, it follows that $s^{I I}<t^{I}$.
} 
$V_{p h}^{I I} \geq V_{p h}^{I}$ is possible. In Appendix A.2, we show that

$$
V_{p h}^{I I} \gtreqless V_{p h}^{I} \quad \Leftrightarrow \quad \frac{\eta_{l}^{2} \eta_{h}\left(\eta_{h}-1\right)}{\left(\eta_{h}-\eta_{l}\right)^{2}+\eta_{l}^{2}\left(\eta_{h}-1\right)} \gtreqless \omega_{p}^{2} .
$$

The left-hand side of the above condition is positive. Thus, for small values of $\omega_{p}$, we have $V_{p h}^{I I}>V_{p h}^{I}$ and an SHI scheme welfare dominates a BI scheme. This is most evident for $\omega_{p}=0$ when the poor earn no income at all. In this case, the median voter prefers to maximize contributions into the welfare schemes, implying $t^{I}=0.5$ and $s^{I I}=0.5$ at the second stage voting game. Even though both welfare schemes are of equal size, type- $p h$ individuals benefit (in expectation) more from SHI than from a lump-sum transfer as the SHI scheme additionally redistributes income to high-risk individuals.

For higher values of $\omega_{p}$, both $t^{I}$ and $s^{I I}$ are smaller but the reduction is larger for $s^{I I}$. Thus, there is a trade-off between income redistribution and subsidized coverage of health care expenditure for $p h$-types. In the extreme case $\omega_{p} \geq \eta_{l}$, we find $t^{I}>0$ and $s^{I I}=0$. Then, the $\mathrm{BI}$ is superior since the SHI scheme finds no political support in the second stage voting game. In sum, higher income inequality as measured by a lower $\omega_{p}$ tends to favor an SHI scheme. Similarly, a high value of $\eta_{l}$ makes the solution with only an SHI scheme more attractive since it increases $s^{I I}$ and brings it closer to the preferred level of $p h$-individuals.

Next, we turn our attention to the case of $s^{I I}=\hat{s}(0)$ in which health care expenditure is fully covered in equilibrium $I I$. Again, we compare the equilibrium, in which only a BI scheme exists (equilibrium $I$ ), with the solution, where solely an SHI scheme is in place (equilibrium II). In Appendix (A.3), we show that

$$
V_{p h}^{I I} \gtreqless V_{p h}^{I} \Leftrightarrow 2 \omega_{p} \sqrt{1-4 \frac{\bar{\pi} M}{\bar{y}}} \gtreqless 1-4 \frac{\pi_{h} M}{\bar{y}}+\omega_{p}^{2} .
$$

Whether SHI dominates a BI scheme now mainly depends on the amount of health care expenditure compared to average income. If health expenditure is relatively small compared to average income, a BI scheme is superior to SHI since redistribution inherent in the SHI scheme is then relatively weak. A high health risk, by contrast, increases the appeal of the SHI solution since the BI scheme comes with relatively expensive private health insurance premiums. If $\omega_{p}=0$, a sufficient condition for the dominance of SHI is $M>\bar{y} /\left(4 \pi_{h}\right)$.

The following proposition provides a summary of our results.

\section{Proposition 2 (Constitutional stage)}

(i) If $\check{s}_{p l}(0)=0\left(\Leftrightarrow \eta_{l}<\omega_{p}\right)$ SHI finds no political support in the second stage voting game and implementation of a BI scheme is always optimal. 
Table 2: Parameter values

\begin{tabular}{cc|c|c|c}
\hline \hline & & \multicolumn{2}{|c|}{ Income } & \\
& & $y_{p}=2$ & $y_{r} \in[2,8]$ & $\Sigma$ \\
\hline \multirow{2}{*}{ Health risk } & $\pi_{l}=0.2$ & $\theta_{p l}=0.42$ & $\theta_{r l}=0.28$ & 0.7 \\
& $\pi_{h} \in[0.2,0.8]$ & $\theta_{p h}=0.18$ & $\theta_{r h}=0.12$ & 0.3 \\
\hline & $\Sigma$ & 0.6 & 0.4 & 1 \\
\hline \hline
\end{tabular}

(ii) If $0<\check{s}_{p l}(0)<\hat{s}(0)$ implying that the restriction that health care expenditure cannot be refunded above 100 percent is non-binding $(\alpha<0)$, implementation of a BI scheme is optimal if

$$
\frac{\eta_{l}^{2} \eta_{h}\left(\eta_{h}-1\right)}{\left(\eta_{h}-\eta_{l}\right)^{2}+\eta_{l}^{2}\left(\eta_{h}-1\right)}<\omega_{p}^{2}
$$

Otherwise, it is best to set up the institutional framework for an SHI scheme only.

(iii) If $\hat{s}(0)<\check{s}_{p l}(0)$ implying that the restriction that health care expenditure cannot be refunded above 100 percent is binding $(\alpha=1)$, implementation of a BI scheme is optimal if

$$
2 \omega_{p} \sqrt{1-4 \frac{\bar{\pi} M}{\bar{y}}} \gtreqless 1-4 \frac{\pi_{h} M}{\bar{y}}+\omega_{p}^{2} .
$$

Otherwise, it is best to set up the institutional framework for an SHI scheme only.

In the following section, we provide a numerical example which illustrates for which parameter constellations (i), (ii), or (iii) in Proposition 2 arise.

\section{Numerical Illustration}

The simulation is based on $M=1$ and on the parameter values as displayed in Table 2. For all possible $\left(\pi_{h}, y_{p}\right)$-combinations, we compute the utility of a poor high-risk agent at $t^{I}$ and $s^{I I}$, and calculate where $V_{p h}$ is highest. Figure 4 illustrates our results.

In the upper left corner $\pi_{h}=\pi_{l}$ and $y_{r}=y_{p}$ and accordingly $\eta_{l}=\omega_{p}=1$. Here, utilities with a BI and an SHI scheme coincide as $t^{I}=s^{I I}=0$. We then successively increase $y_{r}$ (decrease $\omega_{p}$ ) along the $x$-axis and increase $\pi_{h}$ (decrease $\eta_{l}$ ) down the $y$-axis while keeping all other parameters constant.

In the lower left corner, $\eta_{l}$ is small compared to $\omega_{p}$, implying that a BI scheme is superior to SHI since $s^{I I}=0$. In the upper right corner, on the other hand, $\eta_{l}$ is large compared to 


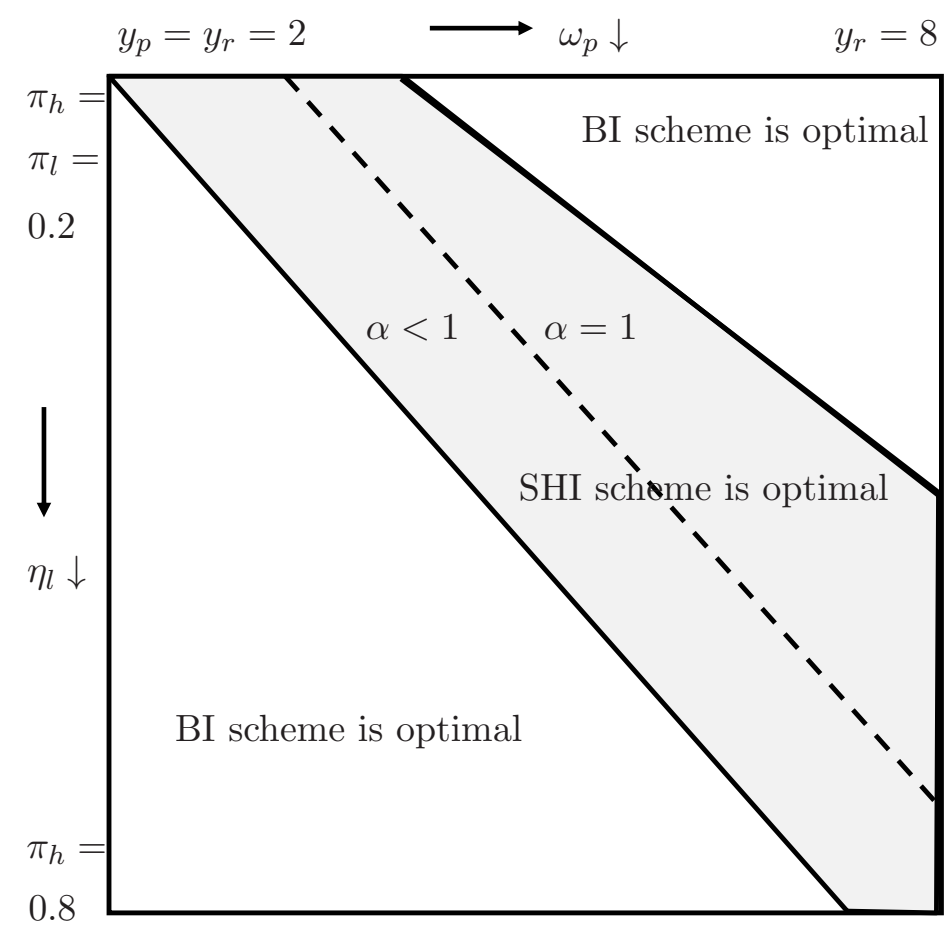

Figure 4: A numerical illustration.

$\omega_{p}$. The SHI rate $s^{I I}$ is then also relatively large. For given health care expenditure, however, this causes the restriction that only up to 100 percent of health care expenditure is publicly financed to be binding and this imposes an upper bound on the redistributive benefits of SHI. A BI scheme dominates an SHI scheme in these cases as well. Within the gray area between the lower left and upper right corner, the utility of the worst-off individuals is maximized if solely an SHI scheme is in place. For these parameter constellations, a positive SHI rate is supported in the political equilibrium. Additionally, the SHI contribution rate is large enough that the targeting advantage inherent in SHI outweighs the benefits of the larger size of the BI scheme. In these cases, society commits at the constitutional stage to implement solely an SHI system and to abandon a BI scheme.

\section{Conclusion}

The idea of a basic income is part of an ongoing debate on the future of the welfare state. For instance, in Germany the adoption of a BI scheme is discussed by all major political parties (Opielka, 2008). Still, neither Germany nor any other country has so far ever introduced a full BI scheme. Instead, countries rely on social insurance schemes.

This paper sheds light on the ramifications of implementing a BI scheme. We followed 
Atkinson's (1996b) proposal and studied the introduction of a BI scheme within two stages. First, at a constitutional stage society chooses behind a veil of ignorance whether an SHI and/or a BI scheme should exist. In a second stage this choice is followed by the specification of the size of the prevailing scheme(s).

Our major finding is that once both welfare schemes are open for debate, only the BI scheme finds political support in the second stage voting game. SHI is fully crowded out. However, behind the veil of ignorance, individuals have an interest in insurance against becoming a highrisk and low-productivity type. Only the SHI scheme can provide both forms of insurance. At the constitutional stage, it can thus be in the interest of society to set up the institutional framework for solely an SHI scheme and to refrain from implementing a BI scheme. We have shown that this is all the more so, the larger the extent of overall health care expenditure compared to average income, and the lower the health inequality compared to income inequality.

A limitation of our analysis is the assumption that individuals can buy full private health insurance coverage at an actuarial fair price. Private health insurance markets may, however, suffer from adverse selection which translates into actuarial unfair premiums and incomplete insurance coverage. This makes an SHI insurance scheme more attractive (see, e.g., Boadway et al. 2006).

The introduction of a BI scheme may have strong labor market consequences. While we have taken into consideration its effect on the tax base in that we have modeled the Laffer curve effect, we have not accounted for any additional income effects a BI scheme might have. Specifically, the provision of a BI may reduce the attractiveness of additional earnings and individuals may withdraw from the labor market. Since no country has ever introduced a BI scheme it is difficult to assess how citizens would change their labor supply in response to a BI. Marx and Peeters (2008) proposed the study of lottery winners who had won a periodically unconditional lifelong income (€1000 per month) to gather empirical information on the labor supply effects of introducing a BI. The results of a pilot study with Belgian "Win for Life" winners point to no extreme consequences of introducing a BI. They found very few changes with regard to quitting work, reducing working time or becoming self-employed. ${ }^{14}$ These possible adverse labor supply effects would also favor an SHI scheme at the constitutional stage.

Throughout this paper we have concentrated solely on efficiency and redistributive aspects of BI and SHI schemes, but have disregarded arguments in support of the philosophical foundations of a BI scheme. For instance, according to Van Parijs (1995) a BI offers each individual the means to lead his life as he pleases and thereby gives meaning to the idea of real freedom. Taking into consideration these other aspects would require a much more complex modeling of individual preferences, for instance, differing views concerning the value of leisure, and that is

\footnotetext{
${ }^{14}$ However, this study has its limitations. In particular, the sample size was rather small (84 winners participated in the survey) and the study only measured short-term effects, but no long-term effects.
} 
left for future research.

\section{Appendix}

\section{A.1 Optimal Solution for $p h$-Type}

The solution to problem (29) is given by the intersection of the reaction functions for $p h$-types, $t_{p h}^{*}(s)$ and $s_{p h}^{*}(t)$, which we derived in Section 3 (see equations (11 and (19)):

$$
\begin{aligned}
& t_{p h}^{*}(s)=\max \left\{0 ; \frac{1-\omega_{p}-\left(1+\eta_{h}\right) s}{2}\right\} \\
& s_{p h}^{*}(t)=\max \left\{0 ; \min \left\{\frac{\eta_{h}-\omega_{p}-\left(1+\eta_{h}\right) t}{2 \eta_{h}} ; \hat{s}(t)\right\}\right\} .
\end{aligned}
$$

First, we consider $\hat{s}(0) \geq \tilde{s}=\left(1-\omega_{p}\right) /\left(1+\eta_{h}\right)$. Then, $t_{p h}^{*}(0)=\left(1-\omega_{p}\right) / 2$ and $s_{p h}^{*}(0)=$ $\left(\eta_{h}-\omega_{p}\right) /\left(2 \eta_{h}\right)$. Additionally, $t_{p h}^{*}(s)=0$ implies $\tilde{s}_{p h}=\left(1-\omega_{p}\right) /\left(1+\eta_{h}\right), s_{p h}^{*}(t)=0$ yields $\tilde{t}_{p h}=\left(\eta_{h}-\omega_{p}\right) /\left(1+\eta_{h}\right)$. We have $t_{p h}^{*}(0)<\tilde{t}_{p h}$ and $s_{p h}^{*}(0)>\tilde{s}$. Thus, $s_{p h}^{*}(t)$ is always above $t_{p h}^{*}(s)$ and the solution is given by

$$
t_{p h}^{\circ}=0 \quad \text { and } \quad s_{p h}^{\circ}=\min \left\{\check{s}_{p h}(0) ; \hat{s}(0)\right\} \quad \text { if } \quad \tilde{s} \leq \hat{s}(0)
$$

If $\hat{s}(0)<\tilde{s}=\left(1-\omega_{p}\right) /\left(1+\eta_{h}\right)$, the reaction functions cross. The optimal solution is given by

$$
t_{p h}^{\circ}>0 \quad \text { and } \quad s_{p h}^{\circ}=\hat{s}\left(t_{p h}^{\circ}\right)>0
$$

and corresponds to the intersection of

$$
\hat{s}(t) \equiv \frac{1-t-\sqrt{(1-t)^{2}-4 \frac{\bar{\pi} M}{\bar{y}}}}{2} \quad \text { and } \quad t_{p h}^{*}(s)=\frac{1-\omega_{p}-\left(1+\eta_{h}\right) s}{2} .
$$

These two equations can be simplified to

$$
\hat{s}(t)=\frac{1-t-\sqrt{(1-t)^{2}-c}}{2} \text { and } t_{p h}^{*}(s)=a-b s
$$

where $a=\frac{1-\omega_{p}}{2}, b=\frac{\left(1+\eta_{h}\right)}{2}$ and $c=4 \frac{\bar{\pi} M}{\bar{y}}$. Thus,

$$
\hat{s}=\frac{1-a+b \hat{s}-\sqrt{(1-a+b \hat{s})^{2}-c}}{2}
$$


Rearranging and simplifying notation, we can write

$$
\begin{aligned}
2 s & =1-a+b s-\sqrt{(1-a+b s)^{2}-c} \\
(1-a+(b-2) s)^{2} & =(1-a+b s)^{2}-c \\
(1-a)^{2}+2(1-a)(b-2) s+(b-2)^{2} s^{2} & =(1-a)^{2}+2(1-a) b s+b^{2} s^{2}-c \\
-4(1-a) s+\left(b^{2}-4 b+4\right) s^{2} & =b^{2} s^{2}-c \\
4(1-b) s^{2}-4(1-a) s+c & =0
\end{aligned}
$$

The solution of the above quadratic equation is given by

$$
s_{1 / 2}=\frac{1-a \pm \sqrt{(1-a)^{2}-(1-b) c}}{2(1-b)}
$$

Substituting in yields

$$
\hat{s}_{p h}^{\circ}=\frac{1+\omega_{p}-\sqrt{\left(1-\omega_{p}\right)^{2}-2\left(1-\eta_{h}\right) 4 \frac{\bar{\pi} M}{\bar{y}}}}{2\left(1-\eta_{h}\right)}
$$

Since denominator is negative, the negative solution applies. For $t_{p h}^{o}$, we obtain

$$
\hat{t}_{p h}^{\circ}=\frac{1-\omega_{p}}{2}-\frac{1-\eta_{h}^{2}}{2}\left(1+\omega_{p}-\sqrt{\left(1-\omega_{p}\right)^{2}-2\left(1-\eta_{h}\right) 4 \frac{\bar{\pi} M}{\bar{y}}}\right) .
$$

\section{A.2 Constitutional Stage: $\alpha<1$}

We first derive the SHI contribution rate $\bar{s}$ which makes $p h$-individuals indifferent between SHI and the BI scheme. From $V_{p h}^{I}=V_{p h}(s=\bar{s}, t=0)$, we obtain

$$
\left(1-\frac{1-\omega_{p}}{2}\right) y_{p}+\frac{1-\omega_{p}}{2}\left(1-\frac{1-\omega_{p}}{2}\right) \bar{y}-\pi_{h} M=(1-\bar{s}) y_{p}+\eta_{h} \bar{s}(1-\bar{s}) \bar{y}-\pi_{h} M .
$$

The above equation reduces to the following quadratic equation

$$
4 \eta_{h} \bar{s}^{2}-4\left(\eta_{h}-\omega_{p}\right) \bar{s}+\left(1-\omega_{p}\right)^{2}=0
$$

of which the solutions are given by

$$
\bar{s}_{1 / 2}=\frac{\eta_{h}-\omega_{p} \pm \sqrt{\left(\eta_{h}-\omega_{p}^{2}\right)\left(\eta_{h}-1\right)}}{2 \eta_{h}} .
$$


The SHI rate must be smaller than $t^{I}$ to equalize utilities (because of the targeting advantage inherent in the SHI scheme). Thus, the relevant solution is

$$
\bar{s} \equiv \bar{s}_{1}=\frac{\eta_{h}-\omega_{p}-\sqrt{\left(\eta_{h}-\omega_{p}^{2}\right)\left(\eta_{h}-1\right)}}{2 \eta_{h}} .
$$

As $\partial V_{p h} / \partial s>0$ for $s \leq\left(\eta_{h}-\omega_{p}\right) /\left(2 \eta_{h}\right)$, we have

$$
V_{p h}^{I I} \lesseqgtr V_{p h}(s=\bar{s}, t=0)=V_{p h}^{I} \quad \Leftrightarrow \quad s^{I I} \lesseqgtr \bar{s} \quad \Leftrightarrow \quad \frac{\eta_{l}^{2} \eta_{h}\left(\eta_{h}-1\right)}{\left(\eta_{h}-\eta_{l}\right)^{2}+\eta_{l}^{2}\left(\eta_{h}-1\right)} \lesseqgtr \omega_{p}^{2} .
$$

\section{A.3 Constitutional Stage: $\alpha=1$}

When $\alpha=1$, then the SHI rate is given by $s^{I I}=\hat{s}(0)=(1-\sqrt{1-4 \bar{\pi} M / \bar{y}}) / 2$. We have $V_{p h}^{I I} \geq V_{p h}^{I}$ if

$$
\begin{aligned}
\left(1-s^{I I}\right) y_{p} & \geq\left(1-\frac{1-\omega_{p}}{2}\right) y_{p}+\frac{1-\omega_{p}}{2}\left(1-\frac{1-\omega_{p}}{2}\right) \bar{y}-\pi_{h} M \\
-4 s^{I I} & \geq \omega_{p}\left(1-\omega_{p}\right)^{2}-4 \frac{\pi_{h} M}{\bar{y}} \\
2 \omega_{p} \sqrt{1-4 \frac{\bar{\pi} M}{\bar{y}}} & \geq 1-4 \frac{\pi_{h} M}{\bar{y}}+\omega_{p}^{2} .
\end{aligned}
$$

\section{References}

[1] Atkinson, A.B., "The Case for a Partcipation Income," The Political Quarterly, 1996a, $67(1), 67-70$.

[2] Atkinson, A.B., Public Economics in Action: The Basic Income/Flat Tax Proposal, 1996b, Oxford University Press.

[3] Boadway, R., M. Leite-Monteiro, M. Marchand, and P. Pestieau, "Social Insurance and Redistribution with Moral Hazard and Adverse Selection," Scandinavian Journal of Economics, 2006, 108 (2), 279-298.

[4] Casamatta, G., H. Cremer, and P. Pestieau, "Political Sustainability and the Design of Social Insurance," Journal of Public Economics, 2000, 75 (3), 686-712.

[5] Clark, C.M., and C. Kavanagh, "Basic Income, Inequality, and Unemployment: Rethinking the Linkage between Work and Welfare,", Journal of Economic Issues, 1996, 30 (2), 399-406. 
[6] Conde Ruiz, J.I., and P. Profeta, "The Redistributive Design of Social Security Systems," The Economic Journal, 2007, 117 (520), 2157-2169.

[7] Friedman, M., Capitalism and Freedom, 1962, Chicago University Press, Chicago.

[8] Galasso, V., "Postponing Retirement: The Political Effect of Aging," Journal of Public Economics, 2008, 92 (10), 2157-2169.

[9] Galasso, V., and P. Profeta, "How Does Ageing Affect the Welfare State?," European Journal of Political Economy, 2007, 23, 554-563.

[10] Kifmann, M., "Health Insurance in a Democracy: Why is It Public and Why are Premiums Income Related?," Public Choice, 2005, 124 (3)-(4), 283-308.

[11] Marx, A., and H. Peeters, "An Unconditional Basic Income and Labor Supply: Results from a Pilot Study of Lottery Winners," The Journal of Socio-Economics, 2008, 37, 16361659.

[12] Meltzer, A.H., and S.F. Richard, "A Rational Theory of the Size of the Government," Journal of Political Economy, 1981, 89 (5), 914-927.

[13] Mossin, J., "Aspects of Rational Insurance Purchasing," Journal of Political Economy, 1968, 76 (4), 553-568.

[14] Nuscheler, R., and K. Roeder, "The Political Economy of Long-Term Care," European Economic Review, 2013, 62, 154-173.

[15] Opielka, M, "The Likelihood of a Basic Income in Germany," International Social Security, 2008, 61 (3), 73-94.

[16] Pauly, M.V., and B. Herring, "Risk Pooling and Regulation: Policy and Reality in Today's Individual Health Insurance Market," Health Affairs, 2007, 26, 770-779.

[17] Rawls, J., A Theory of Justice, 1971, Harvard University Press, Cambridge MA.

[18] Saltman, R.B., R. Busse, and J. Figueras, Social Health Insurance Systems in Western European Countries, 2004, Open University Press.

[19] Shepsle, K., "Institutional Arrangements and Euqilibrium in Multidimensional Voting Models," American Journal of Political Science, 1979, 23 (1), 27-59.

[20] Tobin, J., J.A. Pechman, and P.M. Mieszkowski, "Is a Negative Income Tax Practical?," Yale Law Journal, 1967, r7, 1-27. 
[21] Van Parijs, P., "Why Surfers Should be Fed: The Liberal Case for an Unconditional Basic Income," Philosophy 85 Public Affairs, 1991, 20 (2), 101-131.

[22] Van Parijs, P., "Competing Justifications of a Basic Income,"in: Introduction to Arguing for Basic Income, 1992, Ed.: P. Van Parijs, London: Verso, 3-43.

[23] Van Parijs, P., "Real Freedom for All. What (if Anything) Can Justify Capitalism?,"1995, Oxford University Press, Oxford.

[24] Van Parijs, P., "Basic Income: A Simple and Powerful Idea for the 21st Century," Politics E Society, 2004, 32, 7-40. 